REVIEWS OF INFECTIOUS DISEASES - VOL. 4, SUPPLEMENT - NOVEMBER-DECEMBER 1982

(C) 1982 by The University of Chicago. All rights reserved. 0162-0886/82/0406-0034\$02.00

\title{
Moxalactam Therapy for Obstetric and Gynecologic Infections
}

Stanley A. Gall, W. Allen Addison, and

Gale B. Hill
Frotn the Departments of Obstetrics and Gynecology and of Microbiology and Immunology, Duke University Medical

Center, Durham, North Carolina

\begin{abstract}
Moxalactam, a new cephalosporin antibiotic with a broad spectrum of activity, was evaluated for safety and therapeutic efficacy in the treatment of genital tract infections in women. Fifty-three patients with postpartum endometritis or acute or chronic pelvic inflammatory disease were treated with $2 \mathrm{~g}$ of moxalactam iv every $8 \mathrm{hr}$, usually for five days or longer. Appropriate cultures of peripheral blood, endometrium, cul-de-sac aspirates, urine, wound, and endocervix (only for Neisseria gonorrhoeae) were performed. Overall, $90.6 \%$ (48 of 53) of the patients were successfully treated with moxalactam $-86.2 \%$ (25 of 29 ) and $95.8 \%$ (23 of 24 ) of the patients with endometritis and pelvic inflammatory disease, respectively. Therapy failed in one of five bacteremic patients with endometritis. Of all the bacteria isolated from appropriate culture sites, $58 \%$ (224 of 383) were anaerobes, with anaerobic gram-negative rods-particularly Bacteroides bivius-and gram-positive cocci being predominant. Of 206 anaerobic strains tested with moxalactam by agar dilution techniques, $82 \%$ (169 of 206) were susceptible (minimal inhibitory concentration [MIC], $\leqslant 8 \mu \mathrm{g} / \mathrm{ml}), 11.6 \%$ (24 of 206) were moderately susceptible (MIC, 16-32 $\mu \mathrm{g} / \mathrm{ml}$ ), and 6.3\% (13 of 206) were resistant (MIC, $\geqslant 64$ $\mu \mathrm{g} / \mathrm{ml}$ ). Among the aerobic isolates, enterococci were uniformly resistant. Thus, moxalactam performed well as a single agent in this open clinical trail for women with infections of the genital tract.
\end{abstract}

It is now widely recognized that most obstetric and gynecologic infections - except for uncomplicated gonorrhea-are of polymicrobial etiology, with anaerobic organisms being predominant [1-8]. An important therapeutic consideration is the resistance of many Bacteroides species, which are the most frequently isolated anaerobes, to many of the antibiotics commonly used for treatment of infections of the genital tract. Currently used therapeutic regimens include $(I)$ penicillin, ampicillin, or cephalothin (or a similar cephalosporin) as single agents with the subsequent addition of an aminoglycoside, often with clindamycin or chloramphenicol, if the patient fails to respond; (2) a high-dose, single-agent, second-generation cephalosporin or

Informed consent was obtained from the patients or their parents or guardians as approved by the Clinical Investigation Committee of the Duke University Medical Center; the guidelines for human experimentation of the U.S. Department of Health and Human Services were followed in the conduct of the clinical research.

We thank Freda Kohan, Quida M. Ayers, and Connie Hughes for their excellent technical assistance.

Please address requests for reprints to Dr. Stanley A. Gall, Department of Obstetrics and Gynecology, P.O. Box 3313 . Duke University Medical Center, Durham, North Carolina 27710 . cephamycin [9-11]; (3) an aminoglycoside in combination with penicillin, clindamycin, or metronidazole $[12,13]$; and (4) even a three-drug combination that includes agents active against enterococci, Neisseria gonorrhoeae, anaerobes, and aerobic gram-negative bacilli. The disadvantage of many current therapeutic approaches is that the drug combinations used are expensive or have potentially serious adverse effects. For example, aminoglycoside antibiotics have problems of ototoxicity and nephrotoxicity and in many instances do not offer a significant therapeutic advantage over other drugs. These agents are not effective against anaerobic bacteria, and cephalosporin-resistant aerobic gram-negative rods such as Pseudomonas aeruginosa, for which treatment with an aminoglycoside might be necessary, are uncommon causes of genital tract infections.

The purpose of the present study was the evaluation of the safety and efficacy of moxalactam, a new $\beta$-lactam agent with a modified cephalosporin nucleus, for the treatment of pelvic infections in women. Moxalactam has a broad spectrum of activity, which includes most aerobic and anaerobic gram-positive organisms, gram-negative aerobes (including many species of Pseudomonas), and anaerobes of the Bacteroidaceae family [14-17]. 


\section{Materials and Methods}

Patients. Patients eligible for moxalactam therapy were hospitalized in the obstetrics and gynecology service at Duke University Medical Center and had one of the following diagnoses: (l) postpartum endometritis with or without pelvic cellulitis or (2) acute or chronic pelvic inflammatory disease (PID). Endometritis was diagnosed in postpartum patients with uterine pain who manifested a temperature elevation to $\geqslant 39 \mathrm{C}$ in the 24-hr postpartum period or $>38 \mathrm{C}$ on two occasions at least $6 \mathrm{hr}$ apart $\geqslant 24 \mathrm{hr}$ after delivery and for whom another source of infection was not deemed solely responsible for signs and symptoms. Purulent discharge was not required for inclusion of the patient in the study. Of the 29 cases of endometritis that could be evaluated, 15 occurred in patients who had undergone cesarean sections, 10 in patients who had had vaginal deliveries, and 4 in patients who had undergone therapeutic abortions. PID was diagnosed in the presence of pelvic pain with a pelvic mass, including a tubo-ovarian complex (palpable or demonstrated by ultrasound), fever, or an elevated white blood cell count. Chronic PID was diagnosed in patients with a history of PID who presented with an acute infection. For each patient laboratory evaluations - a complete blood cell count; urinalysis; SMA 18 automated chemistry profile (hepatic, renal, and electrolyte function); and a platelet count-were obtained prior to inclusion in the study, after $72 \mathrm{hr}$, and on completion of treatment.

Bacteriologic cultures. Appropriate samples were obtained for culture before the initiation of therapy and included (when indicated) peripheral blood, endometrium, cul-de-sac aspirate, urine, wound, and endocervix (for N. gonorrhoeae only). For cultures from the endometrium, the endocervical canal was carefully cleaned with povidoneiodine, and a sterile swab was passed to the uterine fundus. The study protocol called for aspiration of the cul-de-sac in all patients, including those with endometritis and those with a diagnosis of PID with tubo-ovarian masses or complexes. Aspirated fluids were placed in a Port-A-Cul ${ }^{(*)}$ vial (BBL Microbiology Systems, Cockeysville, MD), and swab specimens were collected in an anaerobic specimen collector (Becton Dickinson, Rutherford, $\mathrm{NJ}$ ). These anaerobic transport devices are nonnutritive and therefore reduce the likelihood of bacterial overgrowth. Specimens obtained during the day and evening were immediately delivered to the Anaerobic Microbiology Laboratory at the Medical Center for culture, whereas specimens collected during the night were maintained at room temperature $(\sim 22 \mathrm{C})$ in the transport device and delivered to the laboratory the next morning.

When specimens arrived in the laboratory, a gram stain was prepared, and the specimen was placed in an anaerobic glove box (Coy Laboratory Products, Ann Arbor, MI) for inoculation of media for anaerobic and aerobic culture. The specimen was plated onto anaerobically stored brucella blood agar supplemented with $0.5 \mu \mathrm{g}$ of menadione $/ \mathrm{ml}, 5 \mu \mathrm{g}$ of hemin $/ \mathrm{ml}$, and $5 \%$ defibrinated sheep blood; phenylethyl alcohol agar with the same supplements plus $0.5 \%$ yeast extract; and kanamycin-vancomycin agar to which $75 \mu \mathrm{g}$ of kanamycin $/ \mathrm{ml}$ and $7.5 \mu \mathrm{g}$ of vancomy$\mathrm{cin} / \mathrm{ml}$ were added with the other supplements (including yeast extract) in lysed-blood agar for anaerobic incubation. Plates for aerobic incubation included trypticase soy agar with sheep blood, colistin-nalidixic acid blood agar, McConkey's agar, chocolate agar, and Martin-Lewis agar as needed for detection of $N$. gonorrhoeae. Anaerobic chopped-meat carbohydrate broth (Carr-Scarborough Microbiologicals, Atlanta, GA) was also inoculated. Media for culture of aerobic and facultative organisms (termed aerobes in the present study) were incubated at $35 \mathrm{C}$ in a $\mathrm{CO}_{2}$ incubator; these isolates were identified by standard laboratory procedures. Anaerobic plates and broth were incubated for a total of seven and 14 days, respectively, in an anaerobic glove box at $35 \mathrm{C}$. Anaerobic bacteria were identified by morphology on gram stain, gas-liquid chromatography, and biochemical tests performed with the API 20A system (Analytab Products, Chamblee, GA) with the addition of other tests and media as described by Holdeman et al. [18]. Aerobic and anaerobic isolates were immediately tested for susceptibility to moxalactam and other antibiotics by the disk diffusion technique of Kirby et al. [19] and by the broth-disk method [20], respectively. The MICs of moxalactam were determined by agar-dilution techniques for aerobes [21] and anaerobes [22]. Replicate isolates of a single species from different culture sites in a patient were deleted from the 
MIC tests so that susceptibility data would not be skewed.

Drug administration and evaluation. Moxalactam was administered iv in a 1.0-g dose every 8 $\mathrm{hr}$ to the first seven patients and a $2.0-\mathrm{g}$ dose every $8 \mathrm{hr}$ to the subsequent 58 patients. When a patient was judged to be responding to moxalactam therapy, administration of the antibiotic was usually continued for five or more days. Patients with PID who had completed five or more days of moxalactam therapy and were deemed ready for discharge from the hospital were given prescriptions for $250 \mathrm{mg}$ of oral metronidazole four times a day or $100 \mathrm{mg}$ of doxycycline twice a day for 10 days after discharge. Patients with endometritis who completed moxalactam therapy were discharged without prescriptions for oral antibiotic therapy. If no beneficial response occurred after the initial $48-72 \mathrm{hr}$ of the antibiotic regimen, an alternative antibiotic combination was chosen. Peak and trough concentrations of moxalactam in serum of patients were determined by Eli Lilly and Company (Indianapolis, IN).

The evaluation of efficacy and safety of moxalactam therapy was based on clinical response to the antibiotic, fever index, and observation of any adverse effects-including a laboratory finding of any abnormal hematologic, hepatic, or renal function. A second culdocentesis was not considered justified for documentation of a bacteriologic cure of the infection. Clinical cure was defined as apparent diminution of signs (including fever) and symptoms of infection during the initial 48-72 hr of therapy and, finally, resolution of the infection with no need for addition or substitution of other antibiotics while the patient was hospitalized.

\section{Results}

Clinical response. Sixty-five patients were orginally entered in this study. Initially, moxalactam was administered in a dosage of $1 \mathrm{~g}$ every $8 \mathrm{hr}$; of the first seven patients treated with this regimen, only three demonstrated obviously successful responses. Even though patients in whom therapy apparently failed were given a minimal trial of $\geqslant 48 \mathrm{hr}$ of moxalactam treatment, the clinical response was deemed to be poor, and this dosage schedule was judged to be inadequate. The dosage was increased to $2 \mathrm{~g}$ ofmoxalactam every $8 \mathrm{hr}$, and results for these initial seven patients were
Table 1. Clinical response of women with genital tract infections to moxalactam.

\begin{tabular}{lcc}
$\begin{array}{l}\text { Diagnosis } \\
\text { (no. of patients) }\end{array}$ & $\begin{array}{c}\text { No. of patients } \\
\text { in whom } \\
\text { therapy failed }\end{array}$ & $\begin{array}{c}\text { No. of patients } \\
\text { cured (\%) }\end{array}$ \\
\hline $\begin{array}{l}\text { Endometritis (29) } \\
\begin{array}{l}\text { Pelvic inflammatory } \\
\text { disease (24) }\end{array}\end{array}$ & 4 & $25(86.2)$ \\
\hline
\end{tabular}

NOTE. The patients were given $2 \mathrm{~g}$ of moxalactam every 8 $\mathrm{hr}$ for five or more days. Cure was defined as clinically apparent diminution of signs -including fever - and symptoms of infection during the initial $48-72 \mathrm{hr}$ of therapy and, finally, resolution of the infection without the addition or substitution of other antibiotics.

not included in further analysis. Of the remaining 58 cases, five could not be evaluated because one patient withdrew from the study, two patients received an inadequate drug supply, and two received the wrong schedule of dosage or administration of the drug. Therefore, results of moxalactam therapy were evaluated for a total of 53 patients.

The number of patients diagnosed in each category of infection and their responses to moxalactam therapy are listed in table 1 . Of 29 patients with endometritis, $25(86.2 \%)$ were successfully treated with $2 \mathrm{~g}$ of moxalactam every $8 \mathrm{hr}$. Of the four patients with endometritis in whom therapy with moxalactam was deemed to have failed, two patients initially responded but then developed low-grade elevations in temperature and were found to have wound infections. Another patient with high temperatures and septicemia due to Escherichia coli was given an alternative antibiotic regimen after receiving only $10 \mathrm{~g}$ of moxalactam. This treatment may not represent an adequate trial period. The $E$. coli isolate was sensitive in vitro to the drug. The fourth patient with endometritis who received additional antibiotics manifested a low-grade fever that lasted for several days. The single patient with PID who required additional antibiotics probably had a pelvic abscess at the time therapy was initiated, as evidenced by culdocentesis in which $10 \mathrm{ml}$ of pus was obtained; she subsequently required surgical drainage. Fifteen patients developed endometritis after cesarean section; eight had received cefamandole prophylaxis, four had received cefazolin prophylaxis, and three had received no antimicrobial prophylaxis. Blood cultures were positive for five patients, all of 
whom had endometritis; the isolates were Gaffkya anaerobia, Bacteroides ruminicola subspecies ruminicola, Enterobacter aerogenes (probably from a urinary tract infection), and $E$. coli (from two patients, one of whom was previously mentioned as having not responded to therapy). In addition, two patients had urinary tract infection: both urine and blood were positive for $E$. aerogenes in one patient, and urine was positive for $E$. coli in the other.

The clinical response of patients to moxalactam therapy was also evaluated by calculating the fever index [23], the mean initial and final leukocyte counts, and the mean number of hours in which the patients had an elevated temperature $(>37 \mathrm{C})$ before remaining afebrile continuously for a $12-\mathrm{hr}$ period (defervescence time) (table 2).

Bacteriology of infection. Anaerobes accounted for $58 \%$ (224 of 383) of the bacteria isolated from appropriate culture sites. All 29 patients with endometritis had positive cultures for aerobes and/or anaerobes from blood, cul-de-sac, or endometrium, whereas $72 \%$ (13 of 18) of the patients with PID had positive cul-de-sac cultures. Six of the 24 patients with PID did not have a culde-sac culture because of failure to obtain fluid, patient refusal, or some other reason. Culdocentesis was attempted in every patient with endometritis, with successful aspiration in $26(90 \%)$. Of the 26 successful aspirates, 15 showed significant bacterial growth. Cultures from the endometrium were obtained from all patients with endometritis, and growth occurred in $24(83 \%)$.

The species most frequently isolated in signifi-

Table 2. Parameters of clinical response of women with genital tract infections to moxalactam.

\begin{tabular}{lcc}
\hline Parameter & $\begin{array}{c}\text { Pelvic inflam- } \\
\text { matory disease } \\
(n=24)\end{array}$ & $\begin{array}{c}\text { Endometritis } \\
(n=29)\end{array}$ \\
\hline Fever index (degree [F]-hr) & 38.0 & 60.5 \\
Mean defervescence time $(\mathrm{hr})^{*}$ & 49.4 & 48.9 \\
Mean duration of therapy $(\mathrm{hr})$ & 128 & 124 \\
Mean leukocyte count ${ }^{\dagger}$ & & \\
$\quad$ Initial & 12.6 & 13.8 \\
$\quad$ Final & 6.1 & 8.9 \\
\hline
\end{tabular}

NOTE. Patients were given $2 \mathrm{~g}$ of moxalactam every $8 \mathrm{hr}$ for five or more days.

* Mean no. of hr of elevated temperature $(>37 \mathrm{C}$ ) before the patient remained afebrile continuously for a 12-hr period.

$\dagger$ Mean no. of leukocytes $/ \mathrm{mm}^{3} \times 1,000$.
Table 3. Organisms most frequently isolated in a therapeutic trial of moxalactam in women with genital tract infections.

\begin{tabular}{lc}
\hline Species & $\begin{array}{c}\text { No. of patients } \\
\text { from whom the } \\
\text { organism was isolated }\end{array}$ \\
\hline Bacteroides bivius & 18 \\
Neisseria gonorrhoeae & 18 \\
Peptostreptococcus anaerobius & 12 \\
Gardnerella vaginalis & 9 \\
Mycoplasma hominis & 9 \\
Peptococcus asaccharolyticus & 9 \\
Gaffkya anaerobia & 8 \\
Group B $\beta$-hemolytic streptococci & 6 \\
Bacteroides asaccharolyticus & 6 \\
Bacteroides melaninogenicus & \\
subspecies intermedius & 6 \\
Streptococcus faecalis & 5 \\
Bacteroides capillosus & 5 \\
Bacteroides thetaiotaomicron & 5 \\
Peptococcus magnus & 5 \\
Bacteroides disiens & 4 \\
Bacteroides ruminicola & 4 \\
Escherichia coli & 4 \\
\hline
\end{tabular}

NOTE. Species were included only if growth was present on direct plating of specimens. Unspeciated groups-e.g., diphtheroids (10 patients) - or species isolated from fewer than four patients were not included.

cant numbers from these 53 patients are listed in order of frequency of isolation in table 3 (significant growth refers to the presence of colonies on primary plating media; any organisms isolated only in broth, which might represent only incidental contamination, were disregarded). The frequent occurrence of anaerobic gram-negative rods and gram-positive cocci-which were isolated in significant numbers from $51 \%$ (27 of 53 ) and $43 \%$ (23 of 53), respectively, of all patients - is again apparent. Bacteroides bivius was isolated in significant numbers from $34 \%$ (18 of 53 ) of the patients, whereas Bacteroides fragilis-previously considered to be the primary organism in obstetric and gynecologic infection-was cultured from only $3.7 \%$ (two of 53 ). Aerobic gram-positive cocci were isolated in significant numbers from $41.5 \%$ ( 22 of 53 ) of the patients; group B streptococci and Streptococcus faecalis (enterococci), although found in only a limited number of patients, were the most common species in this group (table 3 ). N. gonorrhoeae was isolated from $34 \%$ (18 of 53) of the patients, all with a diagnosis of PID. Fifteen of the strains of $N$. gonorrhoeae were isolated 
only from the endocervix, but in four of these patients no cul-de-sac material was available for culture. Three additional patients had both endocervical and cul-de-sac cultures positive for $N$. gonorhoeae. Aerobic gram-negative rods were present in significant numbers in $19 \%$ (10 of 53) of the patients, and $E$. coli was the most common isolate.

Of the 206 anaerobic isolates tested against moxalactam by the agar-dilution technique, $82 \%$ (169) were susceptible (MIC, $\leqslant 8 \mu \mathrm{g} / \mathrm{ml}$ ). Anaerobic isolates that were moderately susceptible or resistant are detailed in table 4 . For all the strains of B. bivius isolated during this study, the $\mathrm{MIC}_{50}$ (concentration of drug that inhibited growth of $50 \%$ of the isolates) and the $\mathrm{MIC}_{90}$ (concentration of drug that inhibited growth of $90 \%$ of the isolates) values of moxalactam were $8 \mu \mathrm{g} / \mathrm{ml}$ and 16 $\mu \mathrm{g} / \mathrm{ml}$, respectively; comparable values with penicillin $\mathrm{G}$ were 16 units $/ \mathrm{ml}$ and 64 units $/ \mathrm{ml}$. The $\mathrm{MIC}_{50}$ and $\mathrm{MIC}_{90}$ values of moxalactam for the $B$. fragilis group of organisms were $4 \mu \mathrm{g} / \mathrm{ml}$ and $>64$

Table 4. Anaerobic organisms moderately susceptible or resistant in vitro to moxalactam that were isolated in a therapeutic trial of moxalactam therapy for women with genital tract infections.

\begin{tabular}{|c|c|c|}
\hline \multirow[b]{2}{*}{ Strains (no. tested) } & \multicolumn{2}{|c|}{ No. of strains } \\
\hline & $\begin{array}{l}\text { Moderately } \\
\text { susceptible }\end{array}$ & Resistant \\
\hline \multicolumn{3}{|l|}{ Bacteroides } \\
\hline bivius (24) & 9 & 0 \\
\hline distasonis (2) & 1 & 1 \\
\hline ovatus (4) & 1 & 2 \\
\hline thetaiotaomicron (5) & 0 & 3 \\
\hline ruminicola (4) & 3 & 0 \\
\hline disiens $(7)$ & 2 & 0 \\
\hline capillosus (6) & 2 & 1 \\
\hline species (6) & 1 & 0 \\
\hline Peptostreptococcus anaerobius (22) & 2 & 3 \\
\hline Peptococcus prevotii (4) & 0 & 1 \\
\hline \multicolumn{3}{|l|}{ Streptococcus } \\
\hline constellatus (1) & 1 & 0 \\
\hline morbillorum (1) & 1 & 0 \\
\hline \multicolumn{3}{|l|}{ Clostridium } \\
\hline difficile (1) & 0 & 1 \\
\hline innocuum (1) & 0 & 1 \\
\hline Lactobacillus jensenii (2) & 1 & 0 \\
\hline
\end{tabular}

NOTE. Among the Bacteroides species, B. distasonis, B. ovatus, and B. thetaiotaomicron are all members of the Bacteroides fragilis group. Moderately susceptible was defined as a range in MIC of $16-32 \mu \mathrm{g} / \mathrm{ml}$, and resistant was defined as an MIC of $\geqslant 64 \mu \mathrm{g} / \mathrm{ml}$. $\mu \mathrm{g} / \mathrm{ml}$, compared with values for penicillin $\mathrm{G}$ of 16 units $/ \mathrm{ml}$ and 32 units $/ \mathrm{ml}$. Among the aerobes tested, the only resistant organisms were enterococci (all isolates) and one isolate of Staphylococcus epidermidis.

Drug levels. The median total dose of moxalactam administered iv to 29 patients with endometritis was $26.5 \mathrm{~g}$, whereas the 24 patients with PID received a median total dose of $33.2 \mathrm{~g}$. No adverse reactions, hepatic or renal abnormalities, or neutropenia occurred. Mild thrombocytosis (a $>25 \%$ increase in platelet count) was observed in $85 \%$ of all patients. In no instance did the total platelet count exceed $500,000 / \mathrm{mm}^{3}$.

Serum levels of moxalactam were measured in 43 patients. The average peak serum level following iv infusion was $77.9 \mu \mathrm{g} / \mathrm{ml}$ (range, $31.2-142.3$ $\mu \mathrm{g} / \mathrm{ml}$ ), based on measurements from 53 samples taken within $1 \mathrm{hr}$ of the conclusion of infusion. The average trough serum level was $8.3 \mu \mathrm{g} / \mathrm{ml}$ (range, $2.0-20.8 \mu \mathrm{g} / \mathrm{ml}$ ), based on measurements from 53 samples drawn just prior to the beginning of iv infusion.

\section{Discussion}

Moxalactam is a new $\beta$-lactam antibiotic with a unique substitution of oxygen for sulfur in the cephem nucleus. This compound possesses improved activity as compared with older cephalosporins against anaerobic bacteria (including B. fragilis and B. bivius) and gram-negative aerobes such as Pseudomonas and Enterobacter. In the present study the clinical response to moxalactam therapy of patients with endometritis or PID was encouraging, with an overall clinical cure rate of $90.6 \%$. The antibiotic was well tolerated, and no instances of hepatic, renal, or hematologic abnormalities were detected. The mild thrombocytosis observed in the majority of patients treated with moxalactam created no apparent problem.

Moxalactam appears to have a long serum $t^{1 / 2}$. With the dosage of $2 \mathrm{~g}$ every $8 \mathrm{hr}$, an average trough serum concentration of $8.3 \mu \mathrm{g} / \mathrm{ml}$ was obtained. The mean peak serum concentration of $77.9 \mu \mathrm{g} / \mathrm{ml}$, as determined from samples obtained within $1 \mathrm{hr}$ of the completion of infusion, probably reflected decreasing concentrations of the drug after the true peak since a similar level has been reported for samples obtained immediately after iv infusion of a 1-g dose in normal volunteers [24]. 
The dosage of $2 \mathrm{~g}$ every $8 \mathrm{hr}$, used in the present study, would be expected to produce serum levels that would exceed the MIC for bacteria in the moderately susceptible category $(16-32 \mu \mathrm{g} / \mathrm{ml})$. On this basis, $93.7 \%$ (193 of 206) of the anaerobic strains isolated were susceptible $(\leqslant 32 \mu \mathrm{g} / \mathrm{ml})$. The $\mathrm{MIC}_{90}$ for the $B$. fragilis group of organisms was high $(>64 \mu \mathrm{g} / \mathrm{ml})$, but the isolates of $B$. fragilis (formerly $\boldsymbol{B}$. fragilis subspecies fragilis) were more susceptible $\left(\mathrm{MIC}_{90}, 4 \mu \mathrm{g} / \mathrm{ml}\right)$ than the other former subspecies of the group.

Thirteen resistant anaerobic strains, mostly species of Bacteroides and gram-positive anaerobic cocci, were isolated during this study. Resistant potential pathogens among the aerobic organisms isolated included all strains of enterococci and, in addition, strains of Mycoplasma hominis. A possible correlation between the clinical failure of moxalactam therapy and the isolation of moderately susceptible or resistant organisms was observed for three of the four patients with endometritis whose therapy failed. The patient whose blood culture was positive for $E$. coli (the endometrial and culdocentesis cultures were also positive) also harbored three species of Bacteroides $-B$. bivius, B. ruminicola, and Bacteroides disiens-all with MICs of moxalactam in the moderately susceptible range $(16-32 \mu \mathrm{g} / \mathrm{ml})$. Another patient whose moxalactam therapy failed had a wound infection, and several resistant organisms were isolated from various sites; enterococci and $\mathrm{Myco}$ plasma were both isolated from the wound and endometrial cultures, and a resistant isolate of Peptostreptococcus anaerobius was also isolated from the endometrial culture. Another of the patients with endometritis had a light growth of Mycoplas$m a$ from the endometrial culture. Although the presence of enterococci was possibly related to treatment failure in one of the patients with endometritis, as just discussed, we [12] and others [10] had not previously found a major influence of enterococci in clinical failure with drugs that are inactive against the organism.

Moxalactam was successful in the treatment of all but one of the patients entered in the present study with the diagnosis of PID. These results must be viewed as preliminary, however, since many of the patients apparently had PID due to $N$. gonorrhoeae. Significant growth of mixed aerobes and/or anaerobes was demonstrated in only seven of the 18 patients from whom cultures of $N$. gonorrhoeae were obtained from the cul-desac as well as from the endocervix. The possible influence of mixed aerobes and anaerobes in the pelvic disease of the six patients from whom a culdocentesis culture was not obtained is unknown.

In summary, moxalactam performed well in this open clinical trial with patients with endometritis or PID. The rate of clinical cure with the drug was similar to that previously reported for obstetric infections [25]. The antibacterial spectrum of moxalactam appears to match well the types of aerobic and anaerobic bacteria encountered in pelvic infections, with only a limited number of exceptions. However, more experience with the drug will elucidate whether the increasing resistance among Bacteroides species to the $\beta$-lactam antibiotics and the somewhat reduced activity against gram-positive organisms (compared with that of pencillin or currently approved cephalosporins) will become a therapeutic problem.

\section{References}

1. Parker, R. T., Jones, C. P. Anaerobic pelvic infections and developments in hyperbaric oxygen therapy. Am. J. Obstet. Gynecol. 96:581-645, 1966.

2. Hall, W. L., Sobel, A. I., Jones, C. P., Parker, R. T. Anaerobic postoperative pelvic infections. Obstet. Gynecol. 30:1-7, 1967.

3. Swenson, R. M., Michaelson, T. C., Daly, M. J., Spaulding E. H. Anaerobic bacterial infections of the female genital tract. Obstet. Gynecol. 42:538-541, 1973.

4. Thadepalli, H., Gorbach, S. L., Keith, L. Anaerobic infections of the female genital tract: bacteriologic and therapeutic aspects. Am. J. Obstet. Gynecol. 117:10341040, 1973.

5. Chow, A. W., Marshall, J. R., Guze, L. B. Anaerobic infections of the female genital tract: prospects and perspectives. Obstet. Gynecol. Surv. 30:477-494, 1975.

6. Eschenbach, D. A., Buchanan, T. M., Pollock, H. M., Forsyth, P. S., Alexander, R., Lin, J.-S., Wang, S.-P., Wentworth, B. B., McCormack, W. M., Holmes, K. K. Polymicrobial etiology of acute pelvic inflammatory disease. N. Engl. J. Med. 293:166-171, 1975.

7. Sweet, R. L. Anaerobic infections of the female genital tract. Am. J. Obstet. Gynecol. 122:891-901, 1975.

8. Miller, J. M., Jr., Pupkin, M. J., Hill, G. B. Bacterial colonization of amniotic fluid from intact fetal membranes. Am. J. Obstet. Gynecol. 136:796-804, 1980.

9. Cunningham, F. G., Gilstrap, L. C., III, Kappus, S. S. Treatment of obstetric and gynecologic infections with cefamandole. Am. J. Obstet. Gynecol. 133:602-610, 1979.

10. Sweet, R. L., Ledger, W. J. Cefoxitin: single-agent treatment of mixed acrobic-anacrobic pelvic infections. Obstet. Gynecol. 54:193-198, 1979. 
11. Gall, S. A., Hill, G. B. High-dose cefamandole therapy in obstetric and gynecologic infections. Am. J. Obstet. Gynecol. 137:919-922, 1980.

12. Gall, S. A., Kohan, A. P., Ayers, O. M., Hughes, C. E., Addison, W. A., Hill, G. B. Intravenous metronidazole or clindamycin with tobramycin for therapy of pelvic infections, Obstet. Gynecol. 57:51-58, 1981.

13. DiZerega, G., Yonckura, L., Roy, S., Nakamura, R. M., Ledger, W. J. A comparison of clindamycin-gentamicin and penicillin-gentamicin in the treatment of postcesarean section endomyometritis. Am. J. Obstet. Gynecol. 134:238-242, 1979.

14. Fass, R. J. in vitro activity of LY12795. Antimicrob. Agents Chemother. 16:503-509, 1979.

15. Neu, H. C., Aswapokee, N., Fu, K. P., Aswapokee, P. Antibacterial activity of a new 1-oxa cephalosporin compared with that of other $\beta$-lactam compounds. Antimicrob. Agents Chemother. 16:141-149, 1979.

16. Jones, R. N., Fuchs, P. C., Sommers, H. M., Gavan, T. L., Barry, A. L., Gerlach, E. H. Moxalactam (LY127935), a new semisynthetic 1-oxa- $\beta$-lactam antibiotic with remarkable antimicrobial activity: in vitro comparison with cefamandole and tobramycin. Antimicrob. Agents. Chemother. 17:750-756, 1980.

17. Jorgensen, J. H., Crawford, S. A., Alexander, G. A. Comparison of moxalactam (LY127935) and cefotaxime against anaerobic bacteria. Antimicrob. Agents Chemother. 17:901-904, 1980.

18. Holdeman, L. V., Cato, E. P., Moore, W. E. C. [ed.]. Anaerobe laboratory manual. 4th ed. Virginia Polytechnic Institute and State University, Blacksburg, 1977.
19. Bauer, A. W., Kirby, W. M. M., Sherris, J. C., Turck, M. Antibiotic susceptibility testing by a standardized single disk method. Am. J. Clin. Pathol. 45:493-496, 1966.

20. Wilkins, T. D., Thiel, T. Modified broth-disk method for testing the antibiotic susceptibility of anaerobic bacteria. Antimicrob. Agents Chemother. 3:350-356, 1973.

21. National Committee for Clinical Laboratory Standards. Standard methods for dilution antimicrobial susceptibility tests for bacteria which grow aerobically. National Committee for Clinical Laboratory Standards, Villanova, PA, 1980.

22. National Committee for Clinical Laboratory Standards. Proposed reference dilution procedure for antimicrobic susceptibility testing of anaerobic bacteria. National Committee for Clinical Laboratory Standards, Villanova, Pa., 1980.

23. Ledger, W. J., Kriewall, T. J. The fever index: a quantitative indirect measure of hospital-acquired infections in obstetrics and gynecology. Am. J. Obstet. Gynecol. 115: 514-520, 1973.

24. Parsons, J. N., Romano, J. M., Levison, M. E. Pharmacology of a new 1-oxa- $\beta$-lactam (LY127935) in normal volunteers. Antimicrob. Agents Chemother. 17:226$228,1980$.

25. Gibbs, R. S., Blanco, J. D., Castaneda, Y. S., St. Clair, P. J. Therapy of obstetrical infections with moxalactam. Antimicrob. Agents Chemother. 17:1004-1007, 1980. 